Case Report

\title{
Albendazole in malignant ascites: a case report and literature review on the successes and adverse effects of therapy
}

\author{
Jasmine J. Mui ${ }^{1 *}$, David Morris ${ }^{2}$ \\ ${ }^{1}$ Department of General Surgery, ${ }^{2}$ Peritonectomy Unit, St George Hospital, Gray Street, Kogarah NSW
}

Received: 17 August 2021

Revised: 16 September 2021

Accepted: 18 September 2021

\author{
*Correspondence: \\ Dr. Jasmine J Mui, \\ E-mail: jasmine.jm.mui@gmail.com
}

Copyright: $($ ) the author(s), publisher and licensee Medip Academy. This is an open-access article distributed under the terms of the Creative Commons Attribution Non-Commercial License, which permits unrestricted non-commercial use, distribution, and reproduction in any medium, provided the original work is properly cited.

\begin{abstract}
Malignant ascites places a significant burden on the healthcare system and has a profound effect on patient quality of life. Albendazole (ABZ) is an anti-helminthic agent that has shown to rapidly reduce malignant ascites with minimal side effects in pre-clinical trials. The following case study explores the effect of ABZ on ascitic volume in a patient with malignant ascites secondary to metastatic colorectal adenocarcinoma. The patient is a 54-year-old man who underwent an ultrasound guided ascitic drainage for worsening ascites secondary to hepatic metastases from sigmoid adenocarcinoma. He was commenced on ABZ based on literature demonstrating that ABZ decreases the volume of malignant ascites via the inhibition of vascular endothelial growth factor. Over the next week, the volume of ascitic fluid decreased significantly, however the patient developed neutropenia unresponsive to granulocyte colony stimulating factor likely in the context of impaired liver function. The efficacy of ABZ in this case study as well as in the literature demonstrates the promising potential for use in reducing malignant ascites. However, this case demonstrates the need for caution for use in patients with liver impairment due to changes to first pass metabolism leading to increased circulating drug toxicity and thus, adverse outcomes.
\end{abstract}

Keywords: Albendazole, Malignant ascites, VEGF, Neutropenia

\section{INTRODUCTION}

Malignant ascites places a significant burden on the healthcare system, with up to 28000 hospital beds utilised for in-patient paracentesis per annum. ${ }^{1}$ The effect on patient quality of life is just as profound, with large volume ascites contributing to nausea and vomiting, anorexia, decreased mobility and patient discomfort. ${ }^{2} \mathrm{Up}$ to $50 \%$ of patients with malignancy will develop malignant ascites, and a significant proportion will require regular paracentesis for symptom control, thus increasing the risk of infection. ${ }^{1}$ Albendazole (ABZ) is a benzimidazole carbamate used as an anti-helminthic agent that has shown promising results in pre-clinical trials on rapidly reducing malignant ascites with very minimal side effects. ${ }^{3}$ The following case study explores the effect of $\mathrm{ABZ}$ on ascitic volume in a patient with malignant ascites secondary to metastatic colorectal adenocarcinoma.

\section{CASE REPORT}

The patient is a 54-year-old man admitted to hospital on the 6th of November with 2-3 weeks of worsening ascites secondary to hepatic metastases from sigmoid adenocarcinoma. At the time of cancer diagnosis, the patient had already had liver, lung, and bladder metastases. He had a Hartmann's resection in 2018 and underwent FOLFOX and Avastin therapy for 12 months before trialing FOLFIRI and Cetuximab with no success. He had then had hepatic arterial chemotherapy with 
DEFLEXIFOL in 2019, however had disease progression despite this. At the time of admission, multiple oncologists had deemed him unsuitable for further chemotherapy and he was only for palliative symptom control.

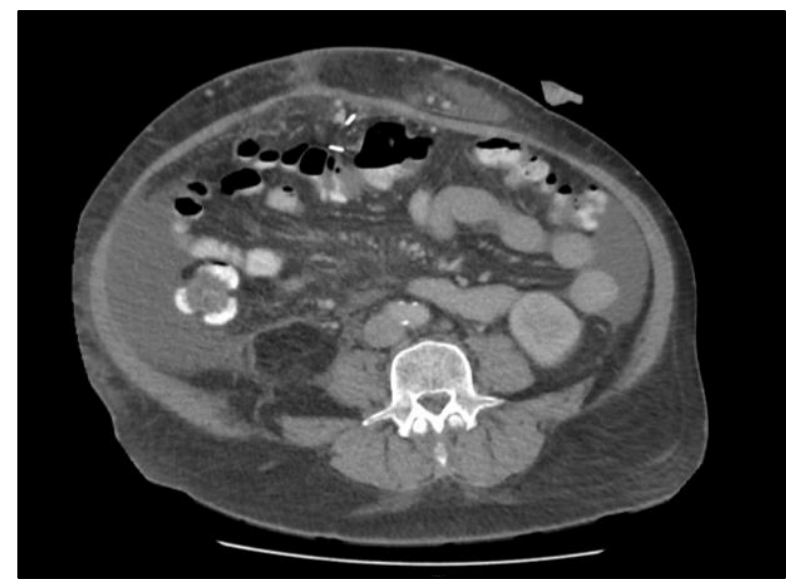

Figure 1: Axial view of CT abdomen demonstraing ascites.

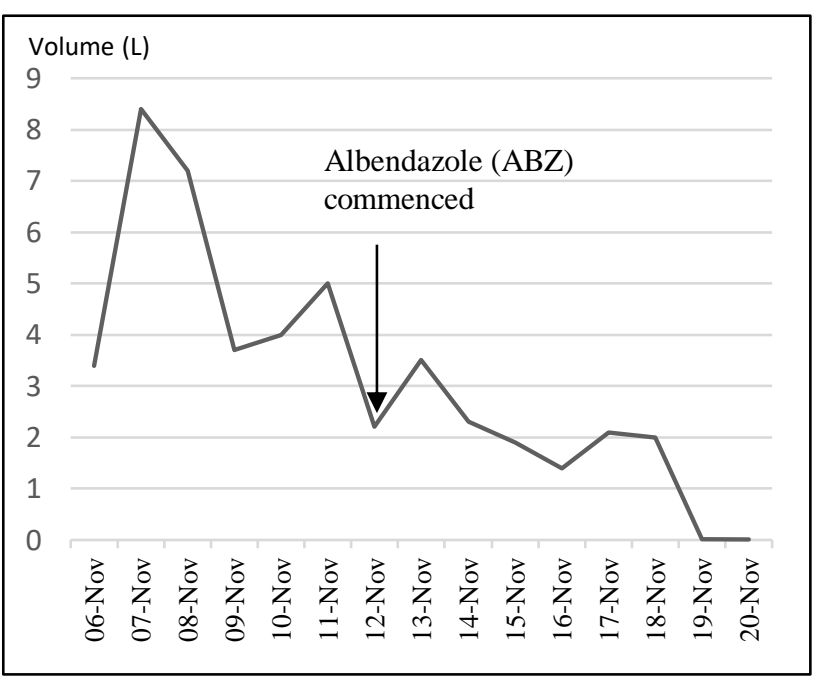

Figure 2: Ascitic volume after commencement of ABZ.

On admission, the patient was haemodynamically stable and afebrile. He was jaundiced with a grossly distended nontender abdomen, and had an active colostomy. His WCC was 10.30, Hb 73, CRP 88, Bilirubin 65, ALP 587, GGT 250, ALT 22, and AST 161. A CT of his abdomen demonstrated ascites and progression of hepatic metastases (Figure 1).

The patient subsequently underwent an ultrasound guided ascitic drainage, with placement of an ascitic drain that produced 3.4litres of fluid upon insertion. A further 8litres was drained over the next 24 hours, with subsequent daily volumes being over 5litres. The patient was commenced on ABZ 400mg BD on the 12th of November based on evidence in the literature suggesting $\mathrm{ABZ}$ has beneficial effects in decreasing the volume of malignant ascites. Over the next week, the volume of ascitic fluid decreased significantly, with only $10-20 \mathrm{mls}$ produced over 24 hours by the 20th of November (Figure 2). A CT abdomen confirmed that there was resolution of abdominal ascites (Figure 3). The ascitic drain was therefore removed on the 21st of November and the patient continued taking ABZ with a plan for discharge.

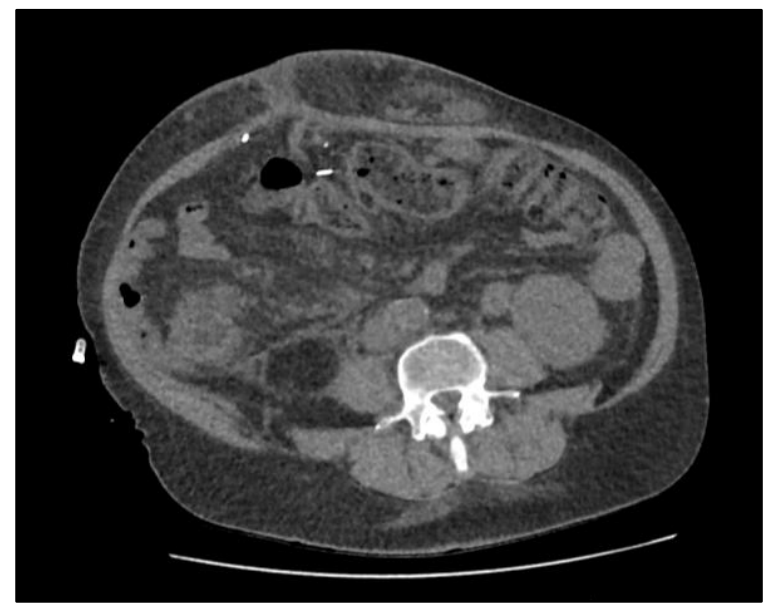

Figure 3: Axial view of CT abdomen demonstraing resolution of ascites.

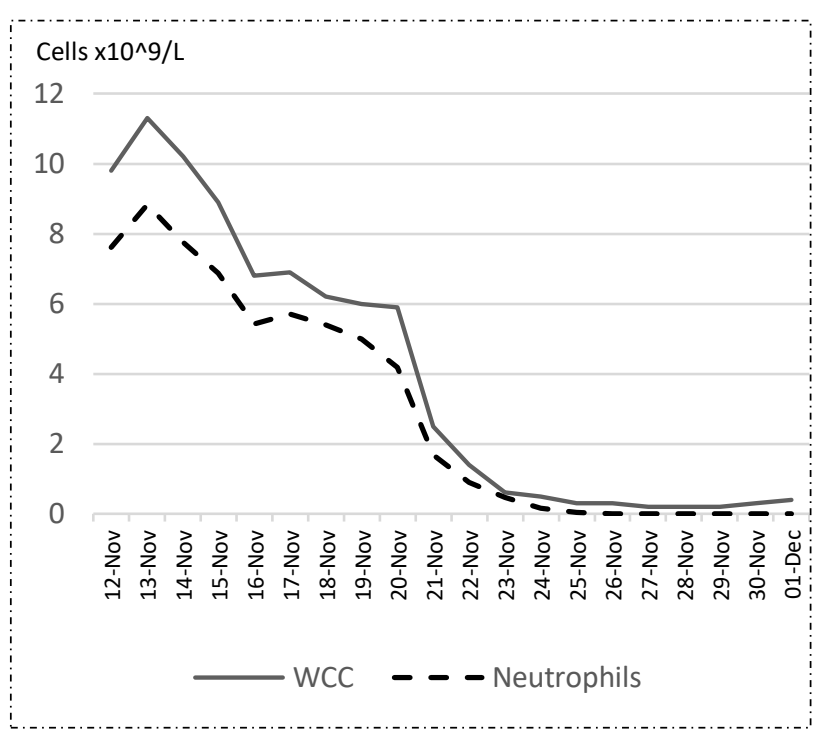

Figure 4: WCC and neutrophils after the commencement of $\mathrm{ABZ}$.

However, over the weekend of the 21-22st of November, it was noted that his WCC and neutrophils were dramatically decreasing, with the WCC falling from 5.9 to 2.5 and neutrophils from 4.19 to 1.69 (Figure 3). A CT scan of the abdomen on the 22st of November did not demonstrate any intra-abdominal cause of pancytopenia and ABZ was ceased on the 23rd of November as the most likely cause of myelosuppression. Despite this, the patient became febrile on the 24th of November and granulocyte colony stimulating factor (GCSF) was commenced on the same day. 


\section{DISCUSSION}

The pathogenesis of malignant ascites is complex and theorised to be linked to increased peritoneal microvascular permeability and neovascularisation via the effect of vascular endothelial growth factor (VEGF). ${ }^{3,4}$ VEGF is a disulfide-bonded dimeric glycoprotein that is produced by tumour stromal cells and binds to endothelial tyrosine kinase receptors VEGFR-1 and VEGFR-2 to increase vascular permeability and protein extravasation, thus inducing the formation of malignant ascites. ${ }^{2,5}$ The relationship between VEGF and ascites is well demonstrated as ascitic volume is directly correlated to VEGF levels. ${ }^{3}$ Therefore, VEGF inhibitors are becoming increasingly prevalent in the symptomatic management of malignant ascites in cases such as this one, where patients have failed other therapies for symptom control.

ABZ is an anti-helminthic used in the treatment of parasites and hydatid cyst disease. It is metabolised by the liver into the active metabolite albendazole sulfoxide, which binds to beta-tubulin proteins to inhibit microtubule polymerisation. ${ }^{3,5-8}$ Microtubules are essential in the formation of mitotic spindles and DNA replication, and inhibition of ABZ leads to the suspension of cell division at metaphase. ${ }^{7,9}$ This has the greatest effect on rapidly dividing cells undergoing mitosis, and thus the antiproliferative nature of $\mathrm{ABZ}$ makes it a potential antitumour agent. Furthermore, ABZ has also been demonstrated to increase reactive oxygen species production, leading to cell cycle arrest and induction of apoptosis.6 Most importantly, ABZ has been shown in the laboratory to reduce VEGF levels, thus reducing the volume of malignant ascites. ${ }^{3,5}$ This is the basis for the use of $\mathrm{ABZ}$ in this case study.

The patient presented with worsening malignant ascites having exhausted all other surgical and oncological avenues for reducing disease burden and symptoms. After the ascitic drain was inserted, large volumes of ascitic fluid were drained daily. However, after commencement of ABZ on day 6 of admission, the amount of fluid declined from 3 litres a day to $20 \mathrm{ml}$ a day within one week. This is consistent with laboratory studies in nude mice, which similarly demonstrated that mice who had ABZ administered three times a week at $150 \mathrm{mg} / \mathrm{kg}$ a day for one month ceased producing macroscopically visible ascites, while their control counterparts still required frequent paracentesis. ${ }^{5}$ Moreover, ABZ has been shown to prevent the development of ascites in nude mice with metastatic cancer if given prior to formation of ascites. ${ }^{3}$ The mechanism of action is due to the downregulation of VEGF mRNA by ABZ, as demonstrated in laboratory studies on VEGF mRNA extracted from malignant ascitic cells. ${ }^{5}$ VEGF mRNA is expressed in a variety of human cancers, such as ovarian, renal, GIT, breast and lung tumours, therefore the potential of ABZ being effective in a myriad of tumour types is promising. ${ }^{5}$ VEGF levels are reported to decrease by up to $80 \%$ in the first 8 hours post administration of ABZ, and ascitic cells numbers, tumour markers and protein levels also downtrend throughout the course of ABZ administration..$^{3,8}$ In addition to reducing ascites, the effects of ABZ on VEGF has other benefits, including inhibition of tumour cell proliferation, tumour cell growth, tumour cell density, and Ki67.,3,5

While effective in decreasing ascitic volume, ABZ had to be ceased in this case due to myelosuppression and resulting febrile neutropenia. This has been previously reported in a pilot study assessing the safety profile of ABZ in patients with colorectal cancer and hepatocellular cancer, where one-third of patients had to cease ABZ due to neutropenia at day 15 after commencement. ${ }^{9}$ Our patient had worsening liver function during ABZ treatment, therefore it is likely that liver impairment impaired the metabolism of ABZ resulting in higher circulating levels and more rapid onset of myelosuppression on day 9 of administration. The worsening liver function was likely multifactorial, due to extensive worsening liver metastasis, biliary obstruction, and ABZ. Deranged liver function tests and myelosuppression are both known adverse effects of microtubule-disruption and thus the variability in hepatic metabolism indicates a need for caution for use in patients with significant liver disease. ${ }^{8}$ Despite this, the adverse effect profile of ABZ has been thoroughly established due to extensive use in hydatid cyst disease. A cyclic regimen of three 28 days courses separated by two-week intervals at $10 \mathrm{mg} / \mathrm{kg} / \mathrm{day}$ is associated with only mild side effects, such as reflux, nausea and vomiting, alopecia and reversible, asymptomatic liver function derangement. ${ }^{10}$ Thus, myelosuppression remains the most significant adverse effect to consider in ABZ use. Administration of $\mathrm{ABZ}$ in cyclic doses may allow for bone marrow recovery between doses, and lower doses in patients with severe liver impairment may prevent inadvertent ABZ toxicity seen with conventional dosing. It should be noted that the efficacy of $\mathrm{ABZ}$ is dose dependent, therefore further research is required to ascertain the most effective doses in patients with liver impairment that minimises myelosuppression. ${ }^{7}$

\section{CONCLUSION}

Targeted therapy for malignant ascites in patients who have failed surgical and systemic chemotherapy would offer significant benefit to quality of life as well as reduce cost to the healthcare system. ABZ has been used extensively in hydatid cyst and helminth disease with very minor side effects, and the efficacy of ABZ in this case study as well as in the literature demonstrates the promising potential for use in reducing malignant ascites. Further data is required to evaluate the safety profile of $\mathrm{ABZ}$ in patients with extensive liver disease due to the impaired drug metabolism and therefore increased toxicity. However, the benefits of ABZ in both reducing ascitic volume as well as decreasing tumour load makes it a worthwhile adjunct to paracentesis in managing malignant ascites. 
Funding: No funding sources

Conflict of interest: None declared

Ethical approval: Not required

\section{REFERENCES}

1. Harding V, Fenu E, Medani H, Shaboodien R, Ngan $\mathrm{S}$, Li HK, et al. Safety, cost-effectiveness and feasibility of daycase paracentesis in the management of malignant ascites with a focus on ovarian cancer. Br J Cancer. 2012;107(6):925-30.

2. Saâda E, Follana P, Peyrade F, Mari V, François E. Pathogenesis and management of refractory malignant ascites. Bull Cancer. 2011;98(6):679-87.

3. Pourgholami MH, Cai ZY, Wang L, Badar S, Links M, Morris DL. Inhibition of Cell Proliferation, Vascular Endothelial Growth Factor and Tumor Growth by Albendazole. Cancer Investigation. 2009;27(2):171-7.

4. Tamsma JT. The Pathogenesis of Malignant Ascites. In: Ceelen WP, editor. Peritoneal Carcinomatosis: A Multidisciplinary Approach. Boston, MA: Springer US; 2007. p. 109-18.

5. Pourgholami HM, Cai YZ, Lu Y, Wang L, Morris LD. Albendazole: a Potent Inhibitor of Vascular Endothelial Growth Factor and Malignant Ascites Formation in OVCAR-3 Tumor-Bearing Nude Mice. Clinical Cancer Research. 2006;12(6):1928-35.

6. Castro LSEPW, Kviecinski MR, Ourique F, Parisotto EB, Grinevicius VMAS, Correia JFG, et al
Albendazole as a promising molecule for tumor control. Redox Biology. 2016;10:90-9.

7. Pourgholami MH, Woon L, Almajd R, Akhter J, Bowery $\mathrm{P}$, Morris DL. In vitro and in vivo suppression of growth of hepatocellular carcinoma cells by albendazole. Cancer Letters. 2001;165(1):43-9.

8. Pourgholami MH, Szwajcer M, Chin M, Liauw W, Seef J, Galettis P, et al. Phase I clinical trial to determine maximum tolerated dose of oral albendazole in patients with advanced cancer. Cancer Chemotherapy and Pharmacology. 2009;65(3):597.

9. Morris DL, Jourdan JL, Pourgholami MH. Pilot Study of Albendazole in Patients with Advanced Malignancy: Effect on Serum Tumor Markers/High Incidence of Neutropenia. Oncology. 2001;61(1):426.

10. Arif SH, Shams ul B, Wani NA, Zargar SA, Wani MA, Tabassum R, et al. Albendazole as an adjuvant to the standard surgical management of hydatid cyst liver. International Journal of Surgery. 2008;6(6):448-51.

Cite this article as: Mui JJ, Morris D. Albendazole in malignant ascites: a case report and literature review on the successes and adverse effects of therapy. Int Surg J 2021;8:3146-9. 